\title{
Arachnoid-Trabeculae Tenting as a Self-retaining Retractor in Resecting Ventrally Located Spinal Meningioma
}

\author{
- Technical Note-
}

\author{
Yuzo YAMAKAWA, Hidenobu OCHIAI, and Tokuro IKEDA
}

Department of Neurosurgery, Miyazaki Prefectural Hospital, Miyazaki

\begin{abstract}
Tenting of the arachnoid with intact trabeculae to facilitate and maintain rotation of the cord during the resection of ventrally or ventrolaterally located spinal meningiomas is proposed. The arachnoidtrabeculae tenting acts as a self-retaining retractor, and prevents overlapping of the cord onto the tumor due to gravity.
\end{abstract}

Key words: spinal meningioma, spinal cord tumor, arachnoid trabecula, self-retaining retractor

\section{Introduction}

The optimal treatment for spinal meningioma is total excision of the tumor with the involved dura. The conventional approach is through an adequate laminectomy and midline dural incision, but a tumor located ventrally or ventrolaterally to the cord cannot always be manipulated easily without pressure ischemia or damage to the cord using conventional self-retaining retractors. ${ }^{2)}$ Other points of operative technique include opening of the dura on the side of the tumor, and preservation of the arachnoid as far as possible with opening only over the tumor. ${ }^{4)}$ Application of traction suture to the dura and direction of traction to the lateral paravertebral muscles or to the tumor other than the cord have been proposed. ${ }^{1,2)}$

The divided dentate ligaments and occasionally the dorsal roots may be used as natural tenting to facilitate and maintain gentle rotation of the cord, especially when resecting ventrally located spinal meningiomas in the cervical to thoracic segments. ${ }^{3,4)}$

We propose tenting of the arachnoid with intact trabeculae together with the dura to achieve gentle and safe rotation of the cord during the procedure of tumor resection.

Received April 10, 1996;

Accepted August 14, 1996

\section{Operative Technique}

The level of the spinal meningioma should be accurately identified. An adequate laminectomy should extend at least one level above and one level below the extent of the tumor. The laminectomy is extended laterally, if necessary adding partial removal of the pedicle on the side of the tumor, to avoid undue manipulation of the spinal cord, except in the case of a dorsally situated tumor.

The dural opening may be in the midline, but should be placed laterally on the side of the tumor in cases with more ventrally located spinal meningiomas. The arachnoid should be preserved and then opened vertically along the dural incision. Dorsally or dorsolaterally located tumors can usually be removed with ease through the midline dural incision using standard microsurgical techniques. Ventrally located tumors require division of the dentate ligaments and occasionally the dorsal roots to facilitate gentle rotation of the cord. At this stage of the operation we preferentially utilize the arachnoid with intact trabeculae for tenting, which maintains the rotated cord during the whole procedure of tumor removal (Fig. 1). This arachnoid tenting together with the dura may prevent overlapping of the cord onto the tumor due to gravity, which is very dangerous on occasion. In this respect the arachnoid-dura tenting works as a self-retaining retractor (Fig. 2). 


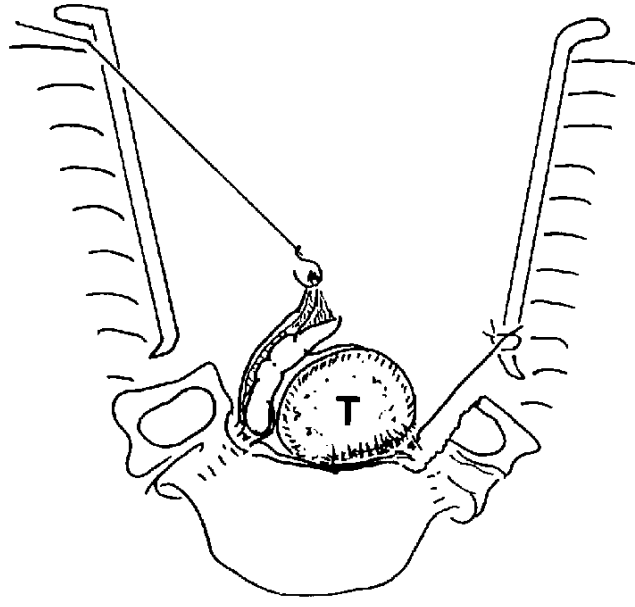

Fig. 1 Schematic drawing depicting the arachnoidtrabeculae self-retaining retractor. To avoid undue manipulation of the spinal cord, the arachnoid-trabeculae should be left intact. The opened arachnoid on the medial side is then tented together with the dura to prevent overlapping of the cord onto the tumor due to gravity. The arachnoid(trabeculae)-dura tenting operates as a self-retaining retractor. T: tumor.

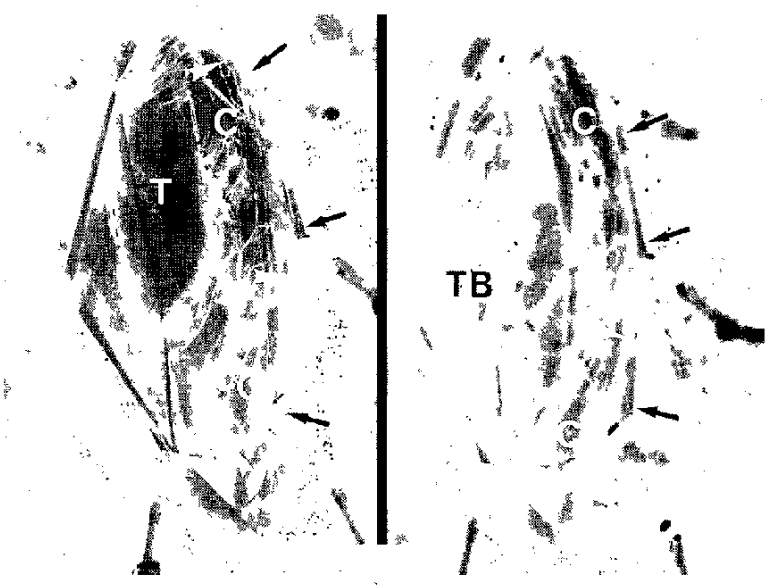

Fig. 2 Intraoperative photograph showing pre- (left) and post-removal (right) stages of the operation. Following the opening of the dura on the side of the tumor, the medial arachnoid (arrowheads) with intact trabeculae is tented together with the dura (arrows). After completion of tumor removal a large space is created and the nerve hung by a hook is a ventral root (right). C: spinal cord, T: tumor, TB: cauterized tumor bed.
Tumor removal should be performed piecemeal with suction, the ultrasonic aspirator, or the carbon dioxide laser, or using the usual bipolar coagulator. Following removal of the tumor, the dura is closed in a watertight fashion. In cases of ventrally placed meningiomas, it may not be possible to close the dural opening adequately, and so a piece of fascia or muscle piece can be inserted into the dural defect and sealed with fibrin glue or an adhesive agent such as aron-alfa.

\section{Discussion}

One danger during the procedure of tumor resection is that the spinal cord may be compressed or rotated repeatedly by spatula manipulation or pulling the divided ligaments to achieve space for working. ${ }^{37}$ Furthermore, the spinal cord is likely to overlap the tumor due to gravity in the prone position, which is routinely used in many institutions to avoid air embolism. Continuous and gentle rotation of the cord can be obtained using the arachnoid tenting as a natural self-retaining retractor. Therefore, the arachnoid-trabeculae should not be cut in the intradural procedure. We have operated on six patients with spinal meningiomas using this technique in the last 4 years, and all patients have recovered well postoperatively. From the minimally invasive operative point of view, this tenting technique may be applicable in managing not only ventrally or ventrolaterally situated spinal meningioma but also spinal neurinoma in similar locations.

\section{References}

1) Connolly RC: Thoracic laminectomy for tumour, including cordotomy, rhizotomy and epidural abscess, in Symon L (ed): Operative Surgery, Fundamental Internationat Techniques. Neurosurgery. London, Butterworth, 1979, pp 472-478

2) Kempe LG: Intradural extramedullary tumor thoracic meningioma, in Kempe LG (ed): Operative Neurosurgery, vol 2. Berlin, Springer-Verlag, 1970, pp 122-125

3) Malik GM, Tomecek FJ, Schmidek HH, Sanders WP: Spinal meningiomas, in Schmidek HH (ed): Meningiomas and Their Surgical Management. Philadelphia, Saunders, 1991, pp 483-489

4) Rose JE: Spinal cord tumors, in Grossmann RG, Hamilton WJ (eds): Principle of Neurosurgery. New York, Raven Press, 1991, pp 359-379

Address reprint requests to: Y. Yamakawa, M.D., Department of Neurosurgery, Miyazaki Prefectural Hospital, 5-30 Kitatakamatsu-cho, Miyazaki 880, Japan.

Neurol Med Chir (Tokyo) 36, December, 1996 